\title{
Relation between Current Transfer Length and Stability of $\mathrm{Fe} / \mathrm{MgB}_{2}$ and $\mathrm{Fe} / \mathrm{Nb} / \mathrm{MgB}_{2}$ Conductors
}

\author{
T. Holúbek, P. Kováč And I. HušEK \\ Institute of Electrical Engineering, Centre of Excellence CENG \\ Slovak Academy of Sciences \\ Dúbravská cesta 9, 84104 Bratislava, Slovak Republic
}

\begin{abstract}
This paper treats and confronts the stability of two different composite $\mathrm{MgB}_{2}$ superconductors and searches for relation between the current transfer length and maximum heat flux that conductor can withstand in operational DC regime. It was found that the iron sheathed wire behave more stably than tape-like conductor with outer iron sheath and inner niobium barrier.
\end{abstract}

PACS numbers: 84.71.Mn

\section{Introduction}

Stable operation of any superconducting device is very crucial topic. Therefore, strong effort is put into the searching for proper design of the device and its operational conditions. Building any specific application such as power cables or superconducting magnets requires research of its basic element from which the device is constructed - superconducting composite conductor. Recently found superconductivity in $\mathrm{MgB}_{2}$ [1] attracts many research groups to investigate stability of composite conductors based on this material [2-4]. Optimizing and improving conductor properties e.g. minimization of heat generation or choice of proper sheath material are very important for further applications. Beside many factors that may influence the conductor stability (conductor shape, number of filaments, filling factor, interface presence, preparation route etc.) we had studied two conductor parameters described below: the current transfer length (CTL) and a maximum heat flux density $p_{\mathrm{t}}$.

The CTL is a conductor-dependent length-scale parameter that essentially determines the length of transfer region where the current is redistributed between superconductor and parallel metallic sheath [5]. It is obtained by measuring potential drop close to the current lead. Anyway, the CTL determines steepness of the potential decay. However, CTL influences indirectly the amount of heat flux 
generated in the transfer volume of the conductor and consequently influences its thermal stability. The heat flux density $p_{\mathrm{t}}$ treated throughout the paper is a power generated in the conductor volume due to non-superconducting current transport at the transition divided by area enclosing this volume. The value $p_{\mathrm{t}}$ is thus maximum heat flux density that conductor can withstand to survive in a stable superconducting state in operational regime. This parameter further determines thermal stability of the conductor.

The aim of this paper is to compare the stability of two different conductors differing by the shape, interface presence and the matrix composition, in the magnetic field region where they exhibit similar critical current densities.

\section{Experiment}

We analyzed two different $\mathrm{MgB}_{2}$ conductors both prepared using in situ technique described in $[6,7]$. The cross-section of the first monofilament wire is square $(1.1 \mathrm{~mm} \times 1.1 \mathrm{~mm})$, where the outer sheath is made of iron (conductor name: S1FeIN). The second one is tape-like monofilament composite $(0.85 \mathrm{~mm} \times 3.45 \mathrm{~mm})$ with inner niobium barrier and the outer iron sheathing (named: T1NbFeIN). Both conductors were measured using 4-point method in different applied magnetic fields while they were immersed in liquid helium bath. The constant ramping rate $\alpha=0.33 \mathrm{~A} / \mathrm{s}$ was kept for all performed measurements. The data of CTL obtained for both samples are taken from paper [8].a

\section{Results and discussion}

Comparable critical current densities of two conductors, S1FeIN and T1NbFeIN, measured in the scale of external magnetic field from 4.5 to $8 \mathrm{~T}$, are presented in Fig. 1a. Bearing in mind all parameters influencing stability that were mentioned in the introduction, similar current densities at $\approx 6 \mathrm{~T}$ permit to compare the total stability of both samples. Right-hand-side Fig. 1b compares theirs CTL in the transport current interval 30-200 A. It was shown by Holúbek et al. that the CTL is independent of magnetic field [8]. Surprisingly, the CTL value of the T1NbFeIN sample is higher than the value of S1FeIN sample over whole transport current interval. This unexpected result is partly explained by the low resistivity of niobium. However, in spite of the absence of chemical reaction layer between $\mathrm{Nb}$ and $\mathrm{MgB}_{2}$, the value of the surface contact resistance is about a factor 10 higher than for the S1FeIN conductor [8]. A possible explanation of this behavior might be the occurrence of the transversal cracks [7] in the $\mathrm{MgB}_{2}$ core due to the very different thermal expansion coefficients of $\mathrm{MgB}_{2}$ and $\mathrm{Nb}$. In the case of S1FeIN conductor, apparent decay of the CTL with increasing transport current is not yet understood [8].

$I-V$ curves recorded above $I_{\mathrm{c}}$ level region for a wide scale of magnetic fields are shown by Fig. 2a. In this current region, the conductor transits from the superconducting into the normal state. The transition is accompanied by 

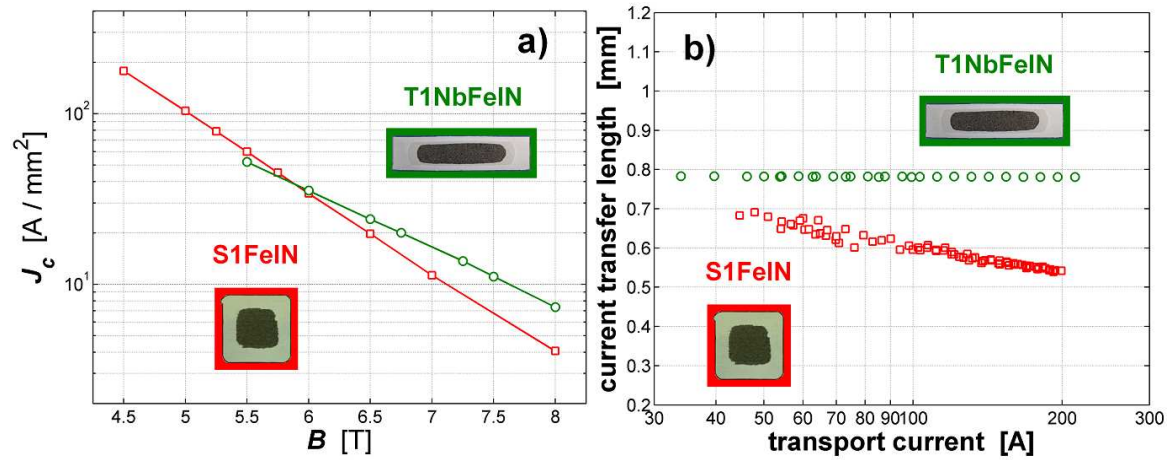

Fig. 1. (a) $J_{\mathrm{c}}(B)$ dependences of analyzed conductors; (b) the current transfer length as a function of transport current $I$.
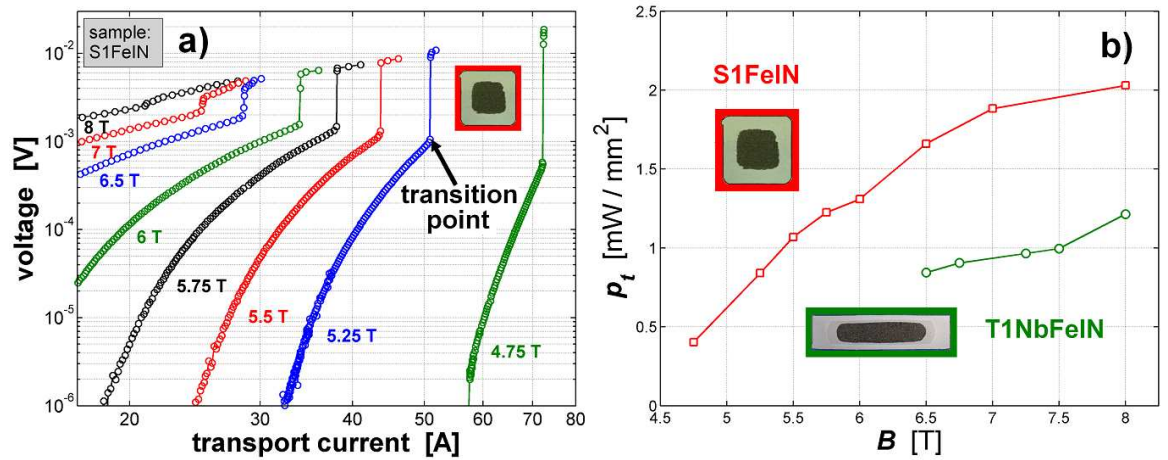

Fig. 2. (a) Hysteretic $I-V$ curves of $\mathrm{MgB}_{2} / \mathrm{Fe}$ conductor recorded in the region far above $I_{\mathrm{c}}$ level. The different magnetic fields were applied; (b) heat flux density at transition point $p_{\mathrm{t}}$ as a function of magnetic field $B$.

the temperature increase caused due to heat dissipation resulting from the nonsuperconducting transport current. The superconductivity vanishes from $\mathrm{MgB}_{2}$ filament at the critical temperature $T_{\mathrm{c}}$. Further, the magnetic field dependence of the heat flux density $p_{\mathrm{t}}$ at the transition point was compared (Fig. 2b). It was found that $p_{\mathrm{t}}$ value decays with increasing magnetic field. An obvious conclusion can be claimed: the higher is the value of $p_{\mathrm{t}}$ the better is the conductor stability. Surprisingly, in spite of higher electrical and thermal conductivity of $\mathrm{Nb}$ in comparison with $\mathrm{Fe}$ and also higher ratio between the effective cooling surface and conductor's volume (which logically improves the thermal stability of conductor), the heat flux $p_{\mathrm{t}}$ of T1NbFeIN conductor is almost twice lower than for S1FeIN conductor in the whole magnetic field region. Together, the CTL and heat flux results indicate that the application of niobium in a direct contact with $\mathrm{MgB}_{2}$ seems not to be very convenient. All the advantageous factors making T1NbFeIN conductor more convenient in comparison with S1FeIN - S1FeIN wire creates resistive in- 
terface $[8,9]$, higher thermal and electrical conductivity of $\mathrm{Nb}$ in comparison with $\mathrm{Fe}$ and the more cooling-convenient shape of T1NbFeIN conductor - do not disprove this statement. One of the possible explanations could dwell in transversal crack occurrence in T1NbFeIN conductor. The cracks causing increased CTL may increase heat generation in the $\mathrm{Nb} / \mathrm{Fe}$ sheathing due to current bypassing created crack and consequently decrease the stability of T1NbFeIN conductor. The large CTL may decrease conductor stability but apparently it is not the only factor in case of T1NbFeIN conductor.

\section{Conclusions}

Thermal stability of two different composite $\mathrm{MgB}_{2}$ conductors was compared and discussed. It was found that the heat flux density at the transition decays with increased magnetic field for both conductors. Almost twice lower transition heat flux values were obtained for the tape-like conductor with niobium barrier when compared with iron sheathed wire. Large current transfer length estimated for conductor with niobium barrier - possible consequence of transversal cracks occurrence - is one of the factors influencing its thermal stability negatively because the heat is generated in the larger volume of composite conductor.

\section{Acknowledgments}

This work was supported by European project HIPERMAG No. NMP3-CT2004-505724.

\section{References}

[1] J. Nagamatsu, N. Nakagawa, T. Muranaka, Y. Zenitani, J. Akimitsu, Nature 410, 63 (2001).

[2] E. Martínez, F. Lera, M. Martínez-Lòpez, Y. Yang, S.I. Schlachter, P. Lezza, P. Kováč, Supercond. Sci. Technol. 19, 143 (2006).

[3] H. Van Weeren, N.C. Van Den Eijnden, W.A.J. Wessel, P. Lezza, S.I. Schlachter, W. Goldacker, M. Dhall, A. Den Ouden, B. Ten Haken, H.H.J. Ten Kate, IEEE Trans. Appl. Supercond. 15, 1667 (2005).

[4] W. Goldacker, S.I. Schlachter, B. Liu, B. Obst, E. Klimenko, Physica C 401, 80 (2004).

[5] M.N. Wilson, Superconducting Magnets, Clarendon Press, Oxford 1986.

[6] P. Kováč, I. Hušek, T. Melišek, V. Štrbík, Supercond. Sci. Technol. 18, 856 (2005).

[7] P. Kováč, I. Hušek, T. Melišek, M. Kulich, V. Štrbík, Supercond. Sci. Technol. 19, 600 (2006).

[8] T. Holúbek, M. Dhall, P. Kováč, Supercond. Sci. Technol. 20, 123 (2007).

[9] T. Holúbek, P. Kováč, T. Melišek, Supercond. Sci. Technol. 18, 1218 (2005). 